\title{
L'élimination de la rougeole en Suisse
}

\section{Christoph Bergera, Barbara Weil ${ }^{b}$}

a Co-responsable de l'unité d'infectiologie et d'hygiène hospitalière, cliniques pédiatriques universitaires, Zurich

b Responsable du département Promotion de la santé et prévention $\mathrm{FMH}$
Correspondance:

Prof. Christoph Berger Universitäts-Kinderkliniken Steinwiesstrasse 75 CH-8032 Zurich Tel. 0442667840 Fax 0442668072
Grâce à la vaccination, la rougeole est devenue rare. Le virus de la rougeole infecte uniquement les êtres humains et se transmet très facilement via la toux et les éternuements. A l'échelle mondiale, la maladie est encore présente principalement en Asie, en Afrique et dans certains pays d'Europe présentant une couverture vaccinale trop faible, à l'instar de la Suisse. Plus de 4400 cas de maladie déclarés, des centaines d'hospitalisations et des coûts supérieurs à 15 millions de francs: entre 2006 et 2009, la Suisse a été confrontée à une épidémie. En 2009, une fillette préalablement en bonne santé est décédée des suites de complications dues à la rougeole. Si l'on renonçait complètement à la vaccination, la Suisse enregistrerait environ 70000 cas de rougeole chaque année et 20 à 30 décès.

Que signifie «éliminer»? En Suisse, la vaccination a permis d'éliminer la poliomyélite et la variole; cette dernière est éradiquée à l'échelle mondiale depuis 1981. La rougeole est réputée éliminée dans un pays ou sur un continent lorsque, sur trois années, on y recense un cas au maximum pour un million d'habitants. Pour ce faire, 95\% de la population doivent être immuns, c'est-à-dire avoir été vaccinés deux fois ou avoir contracté la maladie.

Lorsque la couverture vaccinale atteint $95 \%$, il en résulte une immunité de groupe; autrement dit, le virus de la rougeole ne peut plus se propager et disparaît au sein d'une population donnée. Plusieurs pays scandinaves, l'Australie et l'ensemble de l'Amérique du Nord et du Sud ont montré que cela est possible: grâce à la vaccination, la rougeole y a pratiquement

\section{Figure 1}

Exanthème caractéristique de la rougeole au $3^{\mathrm{e}}$ jour. (Source: Photo: CDC - Centers of Disease Control and Prevention / Dr Heinz F. Eichenwald)

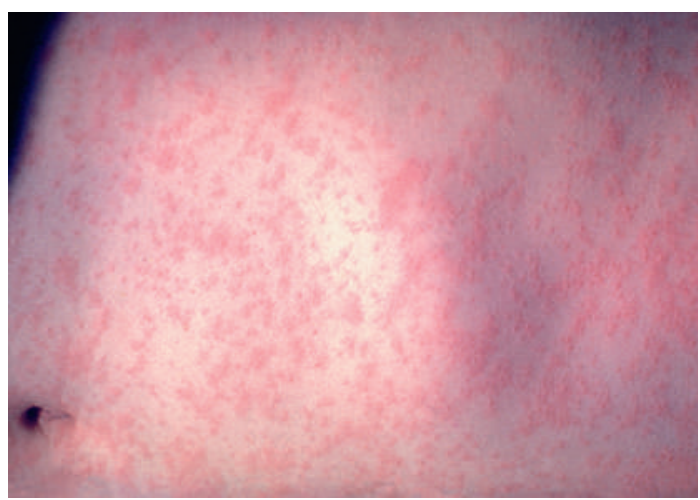

disparu. La Suisse est encore loin d'atteindre ce résultat car, à l'heure actuelle, seuls 85\% des enfants de deux ans ont reçu deux doses de vaccins contre la rougeole. La couverture vaccinale varie fortement d'un canton à l'autre, le canton de Genève se plaçant en tête en 2010, avec un taux de 93\%.

La rougeole est souvent désignée comme étant une «maladie infantile bénigne». Or elle n'est ni bénigne ni uniquement infantile. Les adultes peuvent également en être atteints. La première phase de la maladie commence 7 à 18 jours après l'infection, par des symptômes grippaux et souvent par une conjonctivite. A ce moment-là, les personnes infectées sont d'ores et déjà contagieuses. Nombreux sont les malades qui attribuent ces symptômes à un «refroidissement». Ils contaminent ainsi les personnes non vaccinées de leur entourage et favorisent une propagation très rapide de la rougeole. Quatre jours plus tard commence la deuxième phase, caractérisée par une forte fièvre, un manque d'appétit, une importante sensation de malaise, l'alitement et l'exanthème typique (fig. 1 et 2).

La rougeole peut être à l'origine de complications graves; dans $10 \%$ des cas env., elles requièrent une hospitalisation et peuvent être mortelles. Ce sont ces complications - et non l'éruption cutanée, par ailleurs inoffensive - qui justifient la vaccination. Une otite moyenne, une tracho-bronchite (croup) ou des convulsions fébriles se déclarent dans près de $10 \%$ des cas, une pneumonie dans $5 \%$ des cas. Sur 1000 personnes atteintes d'une rougeole, une souffre d'une encéphalite. Le fait d'être tombé malade ne renforce en aucun cas le système immunitaire, bien au contraire: la rougeole entraîne une leucopénie et une immunosuppression sur plusieurs semaines. Dans de très rares cas, une panencéphalite sclérosante subaiguë (PESS), à l'issue fatale, peut survenir des années après la fin de la maladie comme complications tardive.

La vaccination contre la rougeole est recommandée en Suisse depuis 1976, et la vaccination combinée rougeole/oreillons/rubéole (ROR) depuis 1985. La recommandation applicable à l'heure actuelle est la suivante: une dose de vaccin ROR à 12 mois et une deuxième entre 15 et 24 mois. La vaccination de rattrapage est possible à tout âge et est recommandée à toutes les personnes non immunes nées en 1964 ou plus tard. Deux doses de vaccin ROR offrent en règle générale une protection à vie contre ces maladies. Le recours à ce vaccin combiné vise également à faire disparaître la rubéole. 


\section{Figure 2}

Evolution de la rougeole et propagation. L'apparition de l'exanthème au jour 0 est déterminante pour les mesures à prendre. (Source: service du médecin cantonal, Genève; adapté)

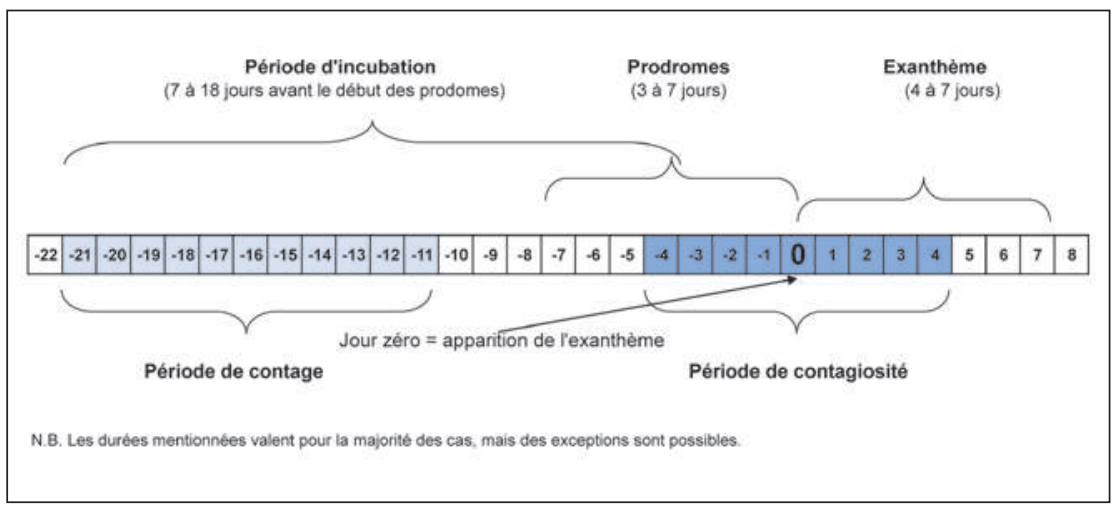

La tolérance au vaccin est très bonne. Depuis les années 70 , plusieurs milliards d'enfants et d'adultes ont été vaccinés contre la rougeole ou ont reçu le vaccin combiné ROR. Une légère éruption cutanée, tout à fait bénigne, peut survenir, voire des convulsions fébriles dans moins de $0,03 \%$ des cas ou une réaction allergique dans moins de $0,001 \%$ des cas. Les femmes se faisant vacciner après la puberté peuvent occasionnellement et passagèrement souffrir de douleurs articulaires. La vaccination est contre-indiquée en cas de grossesse ou d'immunodépression sévère.

\section{La stratégie nationale d'élimination de la rougeole 2011-2015}

«Une Europe sans rougeole d'ici à 2015»: tel est l'objectif visé par les Etats membres de la région européenne de l'Organisation mondiale de la santé (OMS), dont fait partie la Suisse. Pour ce faire, la Confédération, les cantons, différentes associations professionnelles du domaine médical et organisations de la santé ont élaboré ensemble la stratégie nationale pour éliminer la rougeole 2011-2015. Le Conseil fédéral a approuvé sa mise en œuvre en décembre 2011.

Plusieurs mesures sont prévues pour atteindre l'objectif visé; un comité sera notamment institué pour soutenir l'élimination de la rougeole tant sur le plan de la politique que de la communication. La stratégie vise également à ce que 95\% des enfants de deux ans aient reçu deux doses de vaccin. Les structures d'accueil pour enfants devront collaborer, dans toute la mesure du possible, avec un médecin référent. Les parents devront être mieux informés sur la rougeole, sur ses complications et sur la vaccination; de même, ils devront être prévenus à l'avance qu'en cas de flambée de rougeole, les enfants non vaccinés pourraient provisoirement être exclus de la crèche ou de l'école. La stratégie met en outre l'accent sur un accès facilité à la vaccination de rattrapage et sur les offres à bas seuil. Chaque consultation chez le médecin devra constituer l'occasion de vérifier le statut vaccinal et de proposer, le cas échéant, une vaccination de rattrapage. Le vaccin contre la rougeole et le vaccin ROR administrés à titre de rattrapage (consul- rougeole, Berne, état: avril 2013, www.bag.admin. ch/rougeole tation, vaccin et vaccination proprement dite) seront exemptés de la franchise de 2013 à 2015; ainsi, seule la quote-part sera à la charge du patient. La campagne nationale d'information développée par la Confédération et les cantons devra sensibiliser les jeunes et les adultes de moins de 50 ans à la thématique «rougeole et vaccination» et inciter les personnes non immunes à procéder au rattrapage. Il importe de souligner qu'en Suisse la vaccination est et restera volontaire.

Désormais, il est possible de saisir les vaccinations reçues sur le site Internet www.mesvaccins.ch, de faire ainsi vérifier son statut vaccinal et de savoir si d'autres vaccinations sont nécessaires ou si un rappel est recommandé dans le cadre du Plan de vaccination suisse.

Enfin, il s'agira d'améliorer la surveillance épidémiologique de la rougeole. Les cas suspectés de rougeole doivent être rapidement identifiés, signalés au plus vite au service du médecin cantonal et confirmés par une analyse de laboratoire. Les mesures pour contrôler la flambée ne pourront déployer leurs effets qu'à cette condition. Les flambées de rougeole doivent être maîtrisées rapidement et suivant la même procédure au niveau cantonal ${ }^{1}$. Cela fonctionne déjà très bien dans certains cantons, à l'exemple du canton de Genève, qui a pu enrayer une flambée en 2011.

Les médecins jouent un rôle crucial pour ce qui est d'informer leurs patients sur la vaccination, de les inciter à se faire vacciner et d'améliorer la couverture vaccinale. Ils influent grandement sur le comportement de leurs patients en la matière. Ceux d'entre eux qui ne sont pas encore immuns peuvent se prémunir contre la rougeole en entreprenant euxmêmes la démarche: ils donneront ainsi l'exemple et éviteront de propager le virus dans les cabinets médicaux et les hôpitaux.

En outre, les médecins doivent «penser» à proposer le rattrapage, par exemple, à l'occasion d'une consultation pour un rappel de la vaccination contre le tétanos, d'un contrôle gynécologique ou d'une consultation avant un voyage à l'étranger, et contrôler le statut vaccinal du patient, parler ouvertement avec lui des risques et des avantages de la vaccination, répondre à ses questions et inciter les personnes non immunes à se faire administrer le vaccin ROR.

De nombreux cabinets médicaux invitent les parents à faire vacciner leurs enfants aux échéances recommandées. Il existe également, à cet effet, des outils techniques tels que le système Viavac (www. viavac.ch / www.mesvaccins.ch).

Le médecin doit immédiatement déclarer tout cas suspect de rougeole (combinant les trois symptômes suivants: 1. fièvre, 2. exanthème maculo-papuleux et 3. toux, rhinite ou conjonctivite) au service du médecin cantonal et demander une analyse de laboratoire.

Pour plus d'informations: www.bag.admin.ch/ rougeole 